\title{
SCOUT3D - AN UNDERWATER LASER SCANNING SYSTEM FOR MOBILE MAPPING
}

\author{
Michael Bleier, Joschka van der Lucht, Andreas Nüchter
}

\author{
Computer Science VII: Robotics and Telematics, Julius-Maximilians-University Würzburg, Germany \\ \{michael.bleier, joschka.lucht, andreas.nuechter\}@uni-wuerzburg.de
}

\section{Commission II}

KEY WORDS: Underwater Laser Scanning, 3D Reconstruction, Underwater Mapping, Mobile Mapping

\begin{abstract}
:
This paper presents an underwater laser scanning system and GNSS based trajectory estimation system for scanning from a surface vessle in shallow water. The system has an above-the-water and an underwater component. Above-the-water two low-cost multiband GNSS receivers with an antenna baseline of one meter are used for RTK positioning with heading. The full 6-DOF is estimated by fusing the satellite navigation data with a MEMS-based INS. The 3D data is captured in water using a structured light scanner consisting of a low-light underwater camera and a green cross line laser projector. We describe the development of the system and employed hardware components. We show results of scanning a large test object in a water tank acquired by from a tripod with a motorized yaw axis. Additionally, we demonstrate first results of mobile mapping from a floating platform. We evaluate the performance of the system by measuring the 6-DOF trajectory with an external optical tracking system. Additionally, we assess the quality of the created point cloud using reference objects placed in the scene.
\end{abstract}

\section{INTRODUCTION}

In the last decade digital tools are increasingly employed for the acquisition and documentation of archeological sites, in order to achieve higher accuracy and achieve cheaper and more reliable results under economic restrictions. 3D laser scanning is one of the standard optical survey tools for documentation in archeology. The documentation of archeological artifacts underwater is essential. Here it is desirable to document non-destructive and to record data directly in-situ at the underwater site. For example, in the documentation of shipwrecks it is interesting to capture the exact 3D geometry underwater, since bringing parts of the shipwreck to the surface will dry the wooden planks and significantly deform them. This can lead to false conclusions, such as assumptions which parts of the ship hull fit together or where the damage occurred that sunk the ship. Furthermore, archeology is a very cost-sensitive application and accurate 3D documentation ist not always possible due to cost constraints. Unfortunately, the constraints and requirements for each specific site vary and, therefore, not a single sensor system fit all sites and purposes. While digitizing archaeological sites on land as well as artifacts are well understood and standard methods of photogrammetry like 3D modeling from photos or laser scanning can be applied, underwater surveying is still a complex and difficult task. A portable underwater laser scanning system can provide a viable alternative to other documentation methods.

In this paper, we present a structured light underwater laser scanning system for mobile scanning from a surface vessel. The idea is that such a system can be used for 3D scanning in shallow water. Typically, in water we can only cover small areas with an optical scanner due to the limited range. Absorption in water as well as turbidity usually limit the scanning range to a few meters. Therefore, it is necessary to move the scanner in order to digitize larger objects or capture larger areas in water. For example, this can be applied to applications like documentation, searching for artifacts in the water, or sediment analysis.

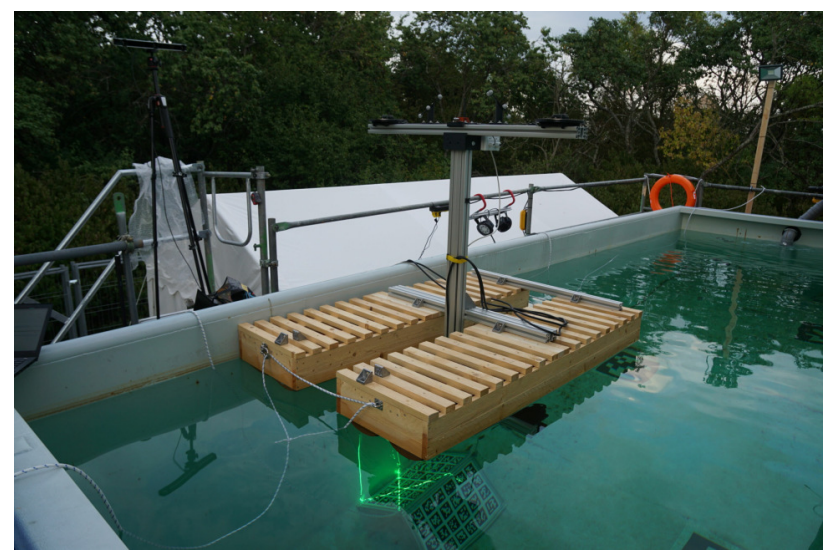

Figure 1. Underwater scanning system mounted to a raft for evaluation in a water tank.

We present the development and construction of a structured light underwater laser scanning system and show first results on applying the system for mobile scanning in the water. The scanning system mounted to a raft is depicted in Fig. 1. For the experiments the raft is moved manually. The structured light scanner is placed in the water pointing downwards. In air a global navigation satellite system (GNSS) real-time kinematic (RTK) positioning system is used to derive precise position data of the scanning system. The 6-DOF trajectory is estimated by fusing satellite navigation data with a MEMS-based inertial navigation system (INS).

\section{RELATED WORK}

Underwater laser scanning systems with larger measurement range often employ high-power line laser projectors. For example, commercial scanners from $2 \mathrm{G}$ Robotics offer a range of up to $10 \mathrm{~m}$ depending on the water conditions (2G Robotics, 2019). 3D scans are typically created by rotating the scan- 

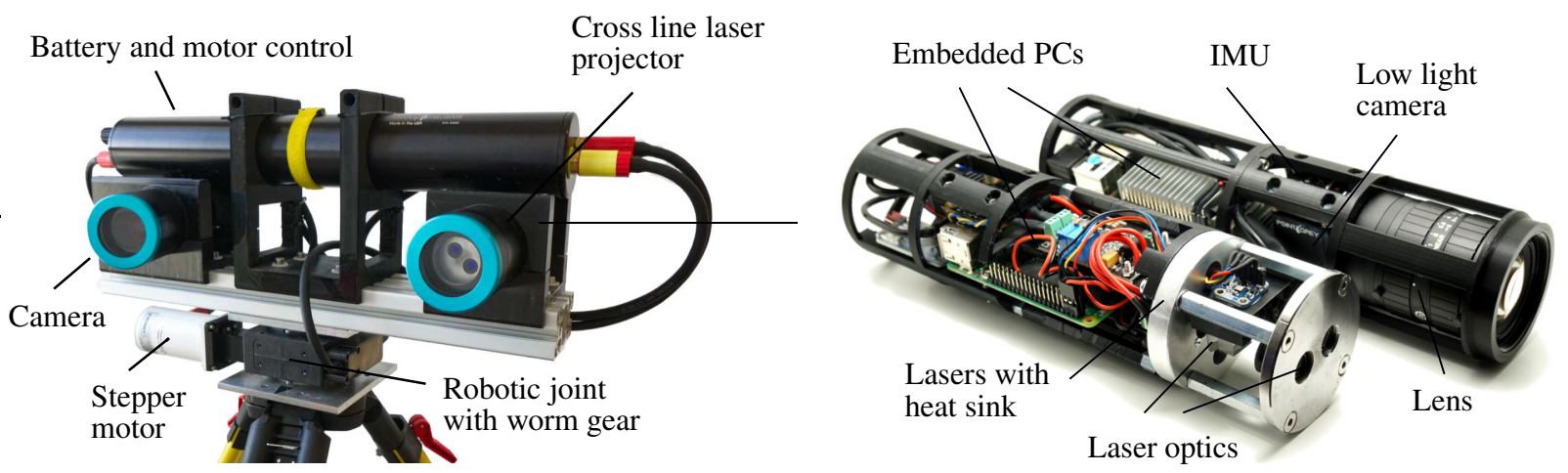

Figure 2. Structured light underwater laser scanner. Left: Scanner with motorized robotic joint mounted on a tripod, right: Detail view of the camera and laser projector assemblies mounted inside the underwater housings.

ner and measuring the movement using rotational encoders or mounting the scanner to a moving platform.

Palomer et al. use a laser projector based on galvanometer scanners (Palomer Vila et al., 2018). This allows to project sweeping laser lines on the scene, which allows to reconstruct the full field of view of the camera. Since in this configuration the laser hits the air-glass and glass-water interface surface at an angle, in water the laser projection cannot be described by a plane. This needs to be modeled explicitly using a physical refraction model. For example, Palomer et al. calibrate the parameters of a cone model to describe the laser projection surface (Palomer et al., 2017).

Another approach is to mount line laser projectors inside a glass cylinder and rotate the line lasers with a motor. This approach is, for example, used in the UX-1 underwater mine exploration robotic system (Martins et al., 2018). In this case the line projectors are aligned manually, such that the projection is perpendicular to the air-glass and glass-water interface surfaces and a straight line is projected. This way the refraction effects are relatively small and are neglected depending on the accuracy requirements. Similarly, the SeaVision subsea 3D laser imaging system developed by Kraken Robotics (Kraken Robotics, 2019) uses rotating red, green and blue line lasers. This enables the system to produced colored scans by evaluating the intensity of the responses of the different laser projectors. Alternatively, diffractive optical elements are used to project multiple lines or a grid for one shot 3D reconstruction (Morinaga et al., 2016, Massot-Campos, Oliver-Codina, 2014). Calibration of these systems is typically achieved using chessboard patterns or 3D calibration fixtures. Some of the parameters can also be determined using self-calibration approaches (Bleier, Nüchter, 2017).

Most of the commercially available underwater laser range sensors are based on laser stripe projection or other forms of structured light. More recently companies started development of time-of-flight (ToF) underwater laser scanners. For example, the company "3D at Depth" developed a commercial underwater LiDAR, which is mounted on a pan-and-tilt unit to create $3 \mathrm{D}$ scans of underwater environments similar to terrestrial pulsed ToF laser scanning (3D at Depth, 2019). A recently proposed scanning system by Mitsubishi uses a dome port with the scanner aligned in the optical center to achieve a wider field of view (Imaki et al., 2017).

Mobile mapping with underwater laser scanners is achieved by using the navigation data of the vehicle. For example, Global
Navigation Satellite System (GNSS) data of a ship (ADUS Deepocean, n.d.) or a combination of acoustic underwater positioning system information, Doppler Velocitiy Log (DVL) and inertial navigation (Martins et al., 2018) is used to measure the vehicle trajectory. Furthermore, Structure-from-Motion (SfM) algorithms are applied to create a trajectory estimate of the scanning system (Duda et al., 2015). With more recently developed underwater laser scanners with high update rate and larger field of view it is possible to incrementally register the point clouds using the Iterative Closest Point (ICP) algorithm. For example, Palomer et al. use a laser scanner for mapping with an AUV. They find an initial coarse alignment using point features and refine the solution using ICP (Palomer et al., 2019).

\section{UNDERWATER LASER SCANNING SYSTEM}

The data presented in this paper was captured with a self-built structured light underwater laser scanning system. The system consists of an above-the-water and an underwater component. Above-the-water a satellite and inertial navigation system is used for trajectory estimation. Below the water surface a custom-built underwater laser scanner is used for 3D data acquisition. Both components are mounted rigidly to the same aluminum profile in order to keep the relative orientation between the trajectory estimation system and the scanner constant during scanning. Since the system uses GNSS for trajectory estimation it can be only applied for mapping in shallow water up to 5-10 meters of water depth .

We choose high power laser line projectors because of the high absorption of light especially in turbid water conditions. Moreover, when scanning in surface water ambient light from the sun is an issue. The laser projection needs to be bright enough, such that sufficient contrast from ambient illumination is achieved. High power lasers and high sensitivity cameras with large dynamic range mitigate these problems to some degree.

We choose a cross line laser pattern because this enables scanning with mostly unrestricted movement. Only the distance between the scanner and the object has to be kept in a certain range, because of field-of-view and focus restrictions. This means that the scanner can reconstruct the surface if the surface vessel is moving in forward direction as well as sideways. Additionally, with multiple laser lines there is the advantage that internal overlap between the created point clouds of the individual line projection is achieved. With a single line we usually only have overlap between the point clouds of individual laps of the vehicle that is carrying the scanner. 


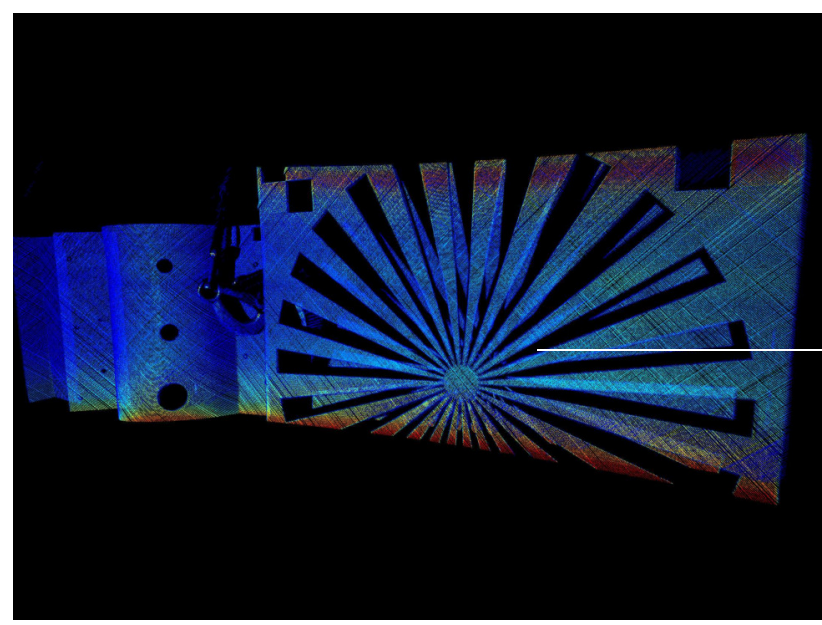

Figure 3. Example scan of the structured light scanner colored by intensity. The scan shows test objects build by IMAWIS $\mathrm{GmbH}$ and Fraunhofer IGP in Rostock. The scan was acquired in the towing tank at the University of Rostock.

The two laser planes are projected at an angle of $45 \mathrm{deg}$ with respect to the vertical camera axis. This way both projected laser lines have approximately the same baseline. However, the resulting baseline is reduced compared to the mounting distance of the camera and laser projector housings.

Fig 3 shows an example point cloud achieved with the proposed system of test objects placed in a water tank. The scan was captured at a distance of approximately $1 \mathrm{~m}$.

\subsection{SCOUT3D Underwater Laser Scanner}

The developed structured light underwater laser scanner consists of two housings with flat port glass windows, one containing the camera and the other one the cross line laser projector. The system is depicted in the left image in Figure 2. The two housings are mounted on a $0.55 \mathrm{~m}$ long aluminum bar. Custom mounts for the housings were manufactured using 3Dprinting. The camera housing is mounted at an angle of $20^{\circ}$ to the bar. All housings include embedded PCs with network interface. The Robot Operating System (ROS) is used as a middleware for sensor interfaces, logging and data processing. All embedded PCs are time-synchronized using Network Time Protocol (NTP) and a pulse-per-second signal. For synchronization of the camera with the laser projector a dedicated trigger pulse signal is used.

The right image in Figure 2 shows the electronics and optics components mounted inside the underwater housings. The camera assembly includes the lens with a focal length of $12.5 \mathrm{~mm}$. The camera is a FLIR Grasshopper3 2.3 Megapixel monochrome camera with a 1/1.2" Sony Pregius IMX249 CMOS sensor and a green band pass filter. The image resolution is $1920 \times 1200$ pixels with $5.86 \mu \mathrm{m}$ pixel size and a maximum framerate of $41 \mathrm{fps}$. For image processing an embedded PC is included in the housing.

The cross line projector is constructed from Powell laser line optics, beam correction prisms and the laser diodes. The lasers are two $1 \mathrm{~W}$ green diode lasers with a wavelength of $525 \mathrm{~nm}$, which are mounted to an aluminum heat sink. The laser output power is controlled by two laser diode drivers, which can be adjusted via PWM signals generated by a microcontroller connected to an embedded PC. The two laser lines project a laser

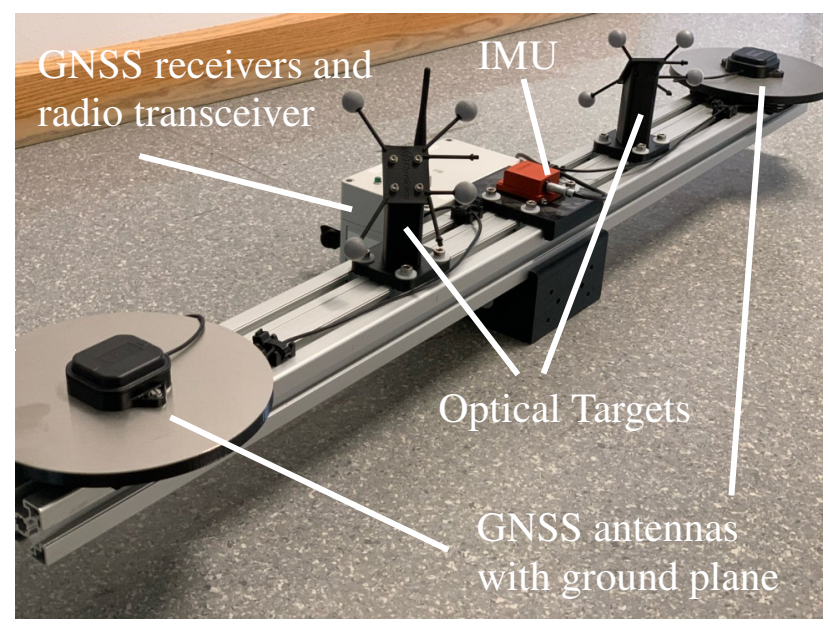

Figure 4. GNSS-INS navigation system with two multiband GNSS antennas and IMU. Correction data from the RTK base station is received via a long range radio transceiver.

cross consisting of two perpendicular lines in the scene. The fan angle of the laser lines is $45 \mathrm{deg}$, which is reduced in water to approximately $32 \mathrm{deg}$. The lasers are fired synchronized to the camera shutter using trigger pulse signals. An alternating firing order of the individual lasers is employed, such that each image captured by the camera includes only one of the two laser lines. An example of the captured 3D point cloud is depicted in Fig. 3.

\subsection{Satellite and Inertial Navigation System}

For mobile scanning, e.g., from a surface vehicle, the underwater laser scanner is combined with a satellite and inertial navigation system. The satellite navigation is based on the cost efficient u-blox ZED-F9P receivers with multiband GNSS patch antennas. The receivers are able to concurrently receive GPS, GLONASS, Galileo and BeiDou satellite navigation data. Two antennas are employed to measure also the heading of the vehicle. Additionally, a Xsens MTi-300 MEMS-based INS system is integrated for measuring the orientation of the system. The INS has a measurement rate of $100 \mathrm{~Hz}$.

The antennas and IMU are mounted on the same aluminum profile, such that the relative orientations are rigid and do not change during transport of the system. The hardware setup is shown in Fig. 4. Additionally, optical ball markers are mounted on the aluminum profile together with the receivers and radio transceiver. This comprises the surface component of the scanning system.

We setup a RTK base station, which sends correction data to the GNSS receivers via a long range radio link. This way real-time RTK positioning and heading data can be computed.

For real-time processing we use the integrated RTK solution of the u-blox receivers, which can provide update rates of up to $10 \mathrm{~Hz}$. Post-processing is done with RTKLIB, which can compute the positioning solution with the full $20 \mathrm{~Hz}$ framerate of the receivers. With this setup centimeter positioning can be achieved depending on the circumstances of the data acquisition, such as occlusion of satellite or multi path effects. For trajectory estimation the position and heading data from satellite navigation is fused with the filtered orientation computed based on the INS data to create a full 6-DOF trajectory. 



Figure 5. Example of estimated GNSS trajectory compared to reference trajectory acquired using an optical tracking system.

Top: Plot of the trajectory, Bottom: Histogram of the 3D position error between the two trajectories.

For reference measurements gray ball markers are attached to the system and the 6-DOF trajectory is measured using an optical tracking system. Fig. 5 shows a trajectory measured using the proposed GNSS RTK system compared to a measurement using an optical tracking system as well as the error histogram. The depicted trajectory has a length of $14 \mathrm{~m}$. The RMSE of the $3 \mathrm{D}$ position error between the RTK trajectory and the reference trajectory is $2.2 \mathrm{~cm}$.

\section{EXPERIMENTS}

For testing we deploy the scanning system in a water test tank built from a container with $40 \mathrm{~m}^{3}$ of water. In the tank we place a test object with a size of $2 \mathrm{~m} \times 2 \mathrm{~m} \times 1 \mathrm{~m}$. The test object is constructed from aluminum profiles and polypropylene pipes. A picture of this structure is depicted in Fig. 6.

Before deployment the pipe structure was scanned in air using a Riegl VZ-400 terrestrial laser scanner. A reference scan of the object was created from eight terrestrial laser scans, which is used for comparison.

We perform static scans of the test structure by mounting the underwater scanner on a tripod and deploying it in the water tank. The setup is shown in the left image of Fig. 6. For mobile scanning experiments we mount the scanner to the raft depicted in Fig. 1.

\subsection{Underwater 3D Scanning Results}

In Fig. 7 are depicted results of the static underwater scans from a tripod. The object is placed at a distance of approximately $2 \mathrm{~m}$ in front of the scanner. Multiple scans from different view points are capture with the underwater scanner by rotating around the motorized yaw axis.

In the top row of Fig. 7 the point cloud results of a scan are show colored by intensity and by height. The intensity image is computed from the brightness of the detected laser points in the camera image.

In the middle row top view and perspective view of a point cloud registered from four underwater scans is shown on the left. The point clouds are colored by height and the poses of the individual poses are visualized by small coordinate systems drawn in the image. On the right the reference scan of the test object is shown. This point cloud is colored by the reflectance values of the terrestrial laser scanner.

In the bottom row the comparison between the reference scan and the underwater point cloud is shown. On the left the reference point cloud is visualized in pink and the underwater point cloud is shown in yellow. The right image shows the registration errors between the reference scan and the underwater scan. While for close range scans of around $1 \mathrm{~m}$ small errors in the range of millimeters are possible to achieve with the system, for this larger object, which encompasses a depth range of approximately $2 \mathrm{~m}$ to $4 \mathrm{~m}$, the errors are in the centimeter range as shown in the error bar on the right side of the image. In this particular case $90 \%$ of the errors are below $2 \mathrm{~cm}$.

\subsection{Underwater Mapping with Mobile Laser Scanner}

For mobile scanning experiments the scanner was mounted to the raft. The floating platform was manually moved above the scan objects. Results of the point clouds created by projecting the individual scans into 3D space along the estimated trajectory from GNSS-INS are shown in Fig. 8.

The conditions in the test tank are not ideal since the lid of the container occludes part of the sky. This means that the GNSS antenna close to the lid provides reduced positioning accuracy. Nevertheless, 3D scans were captured successfully. While the structure of the scanned objects is visible the created point clouds still exhibit coarse errors in the centimeter range. This requires future work to improve the estimated scanner trajectory as well as optimizing the misalignment errors of the system.

\section{CONCLUSIONS}

In this work we presented a structured light underwater laser scanning system and its application for mobile mapping from a surface vessel. First experiments and results of acquiring static and mobile scans in a water tank were described. The software used for controlling the system and sensor data acquisition is available as Open Source at https://github. com/3DTK/SCOUT3D/. Some of the algorithms for data processing and calibration are released in 3DTK - The 3D Toolkit (http://www.threedtk.de/). Using structured light scanners for mobile mapping is interesting because it enables to map larger areas in the water despite the limited measurement range of optical scanners. While the first results are promising future work is required for the optimization of the estimated trajectory of the underwater laser scanner. 

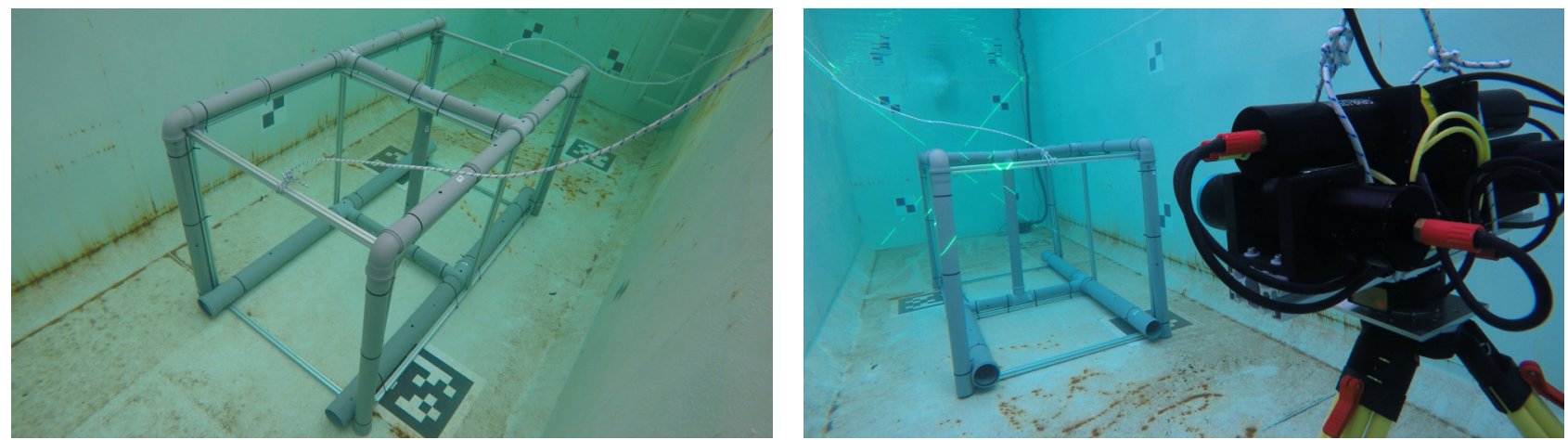

Figure 6. Scanning setup in the test tank. Left: Test structure constructed from aluminum profiles and polypropylene pipes, Right: Underwater laser scanner deployed on a tripod.
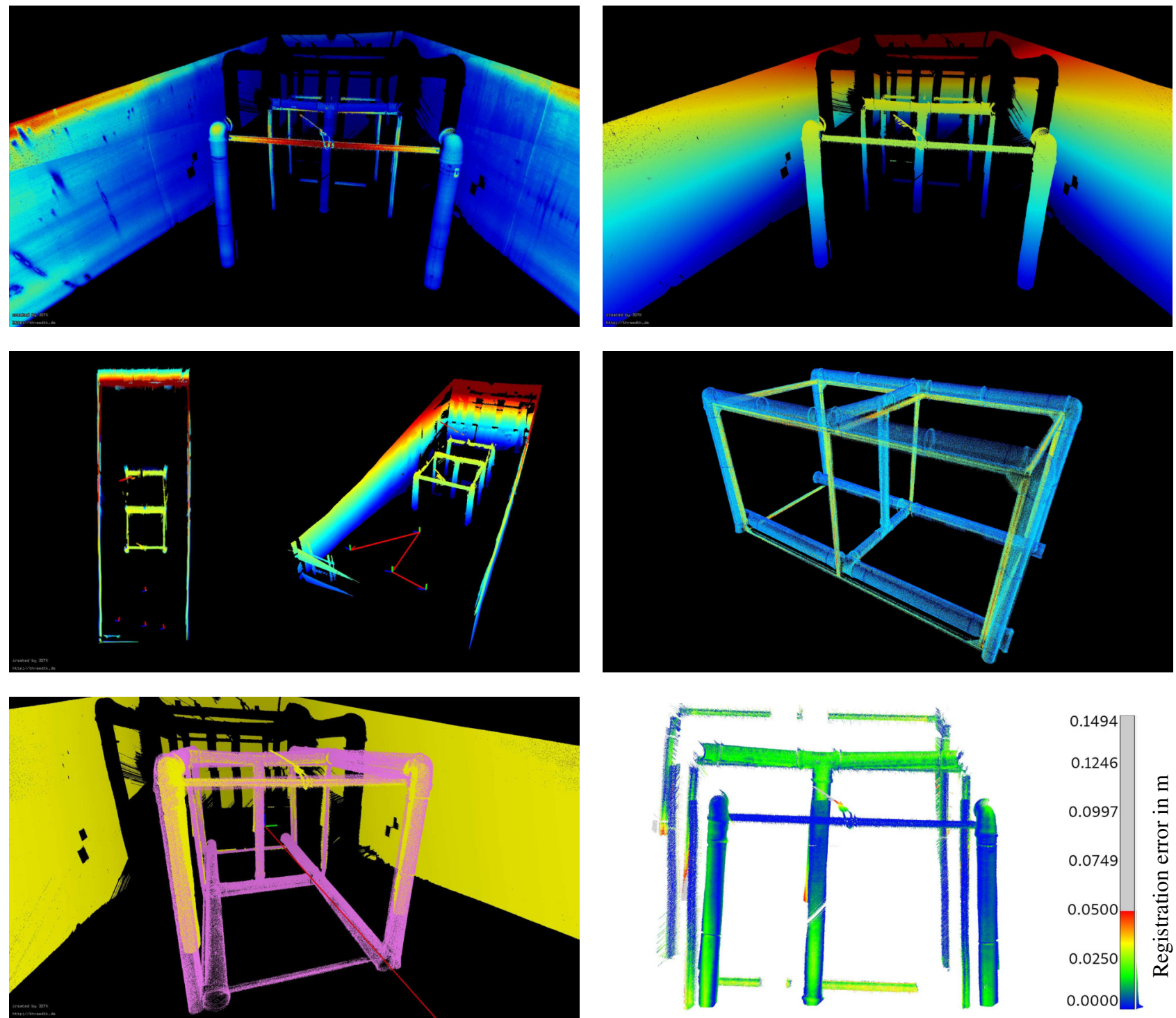

Figure 7. Results of underwater scanning from a tripod. Top left: Point cloud of a scan of the pipe structure colored by intensity, Top right: Same scan colored by height., Middle left: Top view and perspective view of registered point cloud from four scans, Middle right: Reference scan of the structure captured in air before the experiments, Bottom left: Underwater point cloud in yellow and reference scan in pink, Bottom right: Registration error between reference scan and underwater scan. 

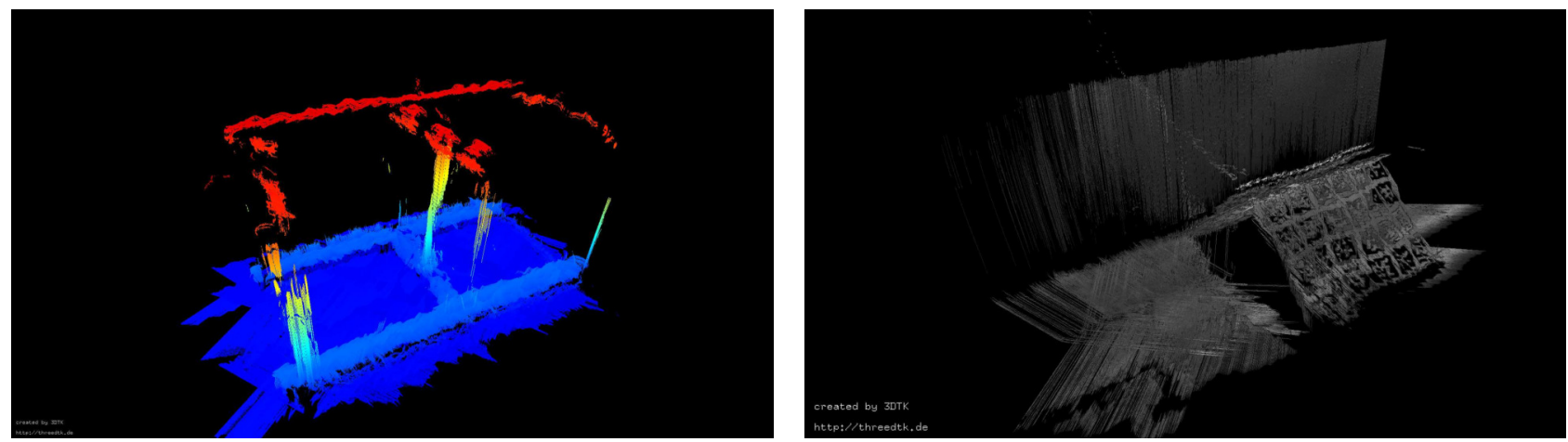

Figure 8. Example of captured point cloud from the floating platform using the GNSS-INS trajectory. Left: Point cloud of the pipe structure colored by height, Right: Point cloud of a calibration target colored by intensity.

\section{ACKNOWLEDGMENT}

This work was funded by the project "Mobile Unterwasserkartierung vom Schiff zur hochpräzisen 3D-Erfassung mittels Laserscannen (MUSCHEL-3D)" under the Central Innovation Programme by the German Federal Ministry for Economic Affairs and Energy (ZIM; No. ZF4117504DF8).

\section{REFERENCES}

2G Robotics, 2019. ULS-500 underwater laser scanner. http://www.2grobotics.com/products/ underwater-laser-scanner-uls-500/. Webpage, accessed October 1, 2019.

3D at Depth, 2019. SL1 subsea lidar. https://www . 3datdepth.com/product/sl1-lidar-laser. Webpage, accessed October 1, 2019.

ADUS Deepocean, n.d. High-quality surveys of man-made structures as an aid to improved decision making. https: //www.video.teledynemarine.com/video/10368962/ high-quality-surveys-of-man-made-structures-as-an. Webpage, accessed October 1, 2019.

Bleier, M., Nüchter, A., 2017. Low-cost 3D Laser Scanning in Air or Water Using Self-calibrating Structured Light. Proceedings of the 7th ISPRS International Workshop 3D-ARCH 2017: "3D Virtual Reconstruction and Visualization of Complex Architectures", ISPRS Archives Photogrammetry and Remote Senssing Spatial Inf. Sci., Volume XLII/W3, Nafplio, Greece, 105-112.

Duda, A., Schwendner, J., Gaudig, C., 2015. SRSL: Monocular self-referenced line structured light. Proceedings of the IEEE/RSJ International Conference on Intelligent Robots and Systems (IROS), 717-722.

Imaki, M., Ochimizu, H., Tsuji, H., Kameyama, S., Saito, T., Ishibashi, S., Yoshida, H., 2017. Underwater three-dimensional imaging laser sensor with 120-deg wide-scanning angle using the combination of a dome lens and coaxial optics. Optical Engineering, 56(3).

Kraken Robotics, 2019. Kraken SeaVision subsea 3D laser imaging system. https://krakenrobotics.com/products/ seavision/. Webpage, accessed October 1, 2019.

Martins, A., Almeida, J., Almeida, C., Dias, A., Dias, N., Aaltonen, J., Heininen, A., Koskinen, K. T., Rossi, C., Dominguez, S., Vörös, C., Henley, S., McLoughlin, M., van Moerkerk, H., Tweedie, J., Bodo, B., Zajzon, N., Silva, E., 2018. UX 1 system design - A robotic system for underwater mining exploration. 2018 IEEE/RSJ International Conference on Intelligent Robots and Systems (IROS), 1494-1500.

Massot-Campos, M., Oliver-Codina, G., 2014. Underwater Laser-based Structured Light System for one-shot 3D reconstruction. Proceedings of IEEE Sensors 2014, 1138-1141.

Morinaga, H., Baba, H., Visentini-Scarzanella, M., Kawasaki, H., Furukawa, R., Sagawa, R., 2016. Underwater Active Oneshot Scan with Static Wave Pattern and Bundle Adjustment. T. Bräunl, B. McCane, M. Rivera, X. Yu (eds), Image and Video Technology, PSIVT 2015, Lecture Notes in Computer Science, 9431, Springer, Cham, 404-418.

Palomer, A., Ridao, P., Ribas, D., 2019. Inspection of an underwater structure using point-cloud SLAM with an AUV and a laser scanner. Journal of Field Robotics.

Palomer, A., Ridao, P., Ribas, D., Forest, J., 2017. Underwater 3D Laser Scanners: The Deformation of the Plane. T. I. Fossen, K. Y. Pettersen, H. Nijmeijer (eds), Sensing and Control for Autonomous Vehicles, Lecture Notes in Control and Information Sciences, 474, Springer, Cham, 73-88.

Palomer Vila, A., Ridao Rodríguez, P., Youakim, D., Ribas Romagós, D., Forest Collado, J., Petillot, Y. R., 2018. 3D Laser Scanner for Underwater Manipulation. Sensors, 18(4). 